\title{
Hybrid Algorithm for Finding Common Elements of the Set of Generalized Equilibrium Problems and the Set of Fixed Point Problems of Strictly Pseudocontractive Mapping
}

\begin{abstract}
Atid Kangtunyakarn
Department of Mathematics, Faculty of Science, King Mongkut's Institute of Technology Ladkrabang, Bangkok 10520, Thailand

Correspondence should be addressed to Atid Kangtunyakarn, beawrock@hotmail.com

Received 8 November 2010; Accepted 14 December 2010

Academic Editor: Qamrul Hasan Ansari

Copyright (C) 2011 Atid Kangtunyakarn. This is an open access article distributed under the Creative Commons Attribution License, which permits unrestricted use, distribution, and reproduction in any medium, provided the original work is properly cited.

The purpose of this paper is to prove the strong convergence theorem for finding a common element of the set of fixed point problems of strictly pseudocontractive mapping in Hilbert spaces and two sets of generalized equilibrium problems by using the hybrid method.
\end{abstract}

\section{Introduction}

Let $C$ be a closed convex subset of a real Hilbert space $H$, and let $F: C \times C \rightarrow \mathbb{R}$ be a bifunction. Recall that the equilibrium problem for a bifunction $F$ is to find $x \in C$ such that

$$
F(x, y) \geq 0, \quad \forall y \in C
$$

The set of solutions of (1.1) is denoted by $\mathrm{EP}(F)$. Given a mapping $T: C \rightarrow H$, let $F(x, y)=$ $\langle T x, y-x\rangle$ for all $x, y \in C$. Then, $z \in \operatorname{EP}(F)$ if and only if $\langle T z, y-z\rangle \geq 0$ for all $y \in C$; that is, $z$ is a solution of the variational inequality. Let $A: C \rightarrow H$ be a nonlinear mapping. The variational inequality problem is to find a $u \in C$ such that

$$
\langle v-u, A u\rangle \geq 0
$$


for all $v \in C$. The set of solutions of the variational inequality is denoted by $\operatorname{VI}(C, A)$. Now, we consider the following generalized equilibrium problem:

$$
\text { Find } z \in C \text { such that } F(z, y)+\langle A z, y-z\rangle \geq 0, \quad \forall y \in C \text {. }
$$

The set of $z \in C$ is denoted by $\operatorname{EP}(F, A)$, that is,

$$
\mathrm{EP}(F, A)=\{z \in C: F(z, y)+\langle A z, y-z\rangle \geq 0, \forall y \in C\}
$$

In the case of $A \equiv 0, \mathrm{EP}(F, A)$ is denoted by $\mathrm{EP}(F)$. In the case of $F \equiv 0, \mathrm{EP}(F, A)$ is also denoted by $\operatorname{VI}(C, A)$. Numerous problems in physics, optimization, variational inequalities, minimax problems, the Nash equilibrium problem in noncooperative games, and economics are reduced to find a solution of (1.3); see, for instance, [1-3].

A mapping $A$ of $C$ into $H$ is called inverse strongly monotone mapping, see [4], if there exists a positive real number $\alpha$ such that

$$
\langle x-y, A x-A y\rangle \geq \alpha\|A x-A y\|^{2}
$$

for all $x, y \in C$. The following definition is well known.

Definition 1.1. A mapping $T: C \rightarrow C$ is said to be a $\mathcal{k}$-strict pseudocontraction if there exists $\kappa \in[0,1)$ such that

$$
\|T x-T y\|^{2} \leq\|x-y\|^{2}+\kappa\|(I-T) x-(I-T) y\|^{2}, \quad \forall x, y \in C .
$$

A mapping $T$ is called nonexpansive if

$$
\|T x-T y\| \leq\|x-y\|
$$

for all $x, y \in C$.

We know that $\kappa$-strict pseudocontraction includes a class of nonexpansive mappings. If $\kappa=1, T$ is said to be a pseudocontractive mapping. $T$ is strong pseudocontraction if there exists a positive constant $\lambda \in(0,1)$ such that $T+\lambda I$ is pseudocontraction. In a real Hilbert space $H,(1.6)$ is equivalent to

$$
\langle T x-T y, x-y\rangle \leq\|x-y\|^{2}-\frac{1-\kappa}{2}\|(I-T) x-(I-T) y\|^{2}, \quad \forall x, y \in D(T) .
$$

$T$ is pseudocontraction if and only if

$$
\langle T x-T y, x-y\rangle \leq\|x-y\|^{2}, \quad \forall x, y \in D(T) .
$$

Then, $T$ is strong pseudocontraction if there exists positive constant $\lambda \in(0,1)$

$$
\langle T x-T y, x-y\rangle \leq(1-\lambda)\|x-y\|^{2}, \quad \forall x, y \in D(T) .
$$


The class of $\kappa$-strict pseudocontractions falls into the one between classes of nonexpansive mappings, and the pseudocontraction mappings, and the class of strong pseudocontraction mappings is independent of the class of $\kappa$-strict pseudocontraction.

We denote by $F(T)$ the set of fixed points of $T$. If $C \subset H$ is bounded, closed, and convex, and $T$ is a nonexpansive mapping of $C$ into itself, then $F(T)$ is nonempty; for instance, see [5]. Browder and Petryshyn [6] show that if a $\mathcal{\kappa}$-strict pseudocontraction $T$ has a fixed point in $C$, then starting with an initial $x_{0} \in C$, the sequence $\left\{x_{n}\right\}$ generated by the recursive formula:

$$
x_{n+1}=\alpha x_{n}+(1-\alpha) T x_{n}
$$

where $\alpha$ is a constant such that $0<\alpha<1$, converges weakly to a fixed point of $T$. Marino and $\mathrm{Xu}$ [7] have extended Browder and Petryshyns above-mentioned result by proving that the sequence $\left\{x_{n}\right\}$ generated by the following Manns algorithm [8]:

$$
x_{n+1}=\alpha_{n} x_{n}+\left(1-\alpha_{n}\right) T x_{n}
$$

converges weakly to a fixed point of $T$ provided the control sequence $\left\{\alpha_{n}\right\}_{n=0}^{\infty}$ satisfies the conditions that $\mathcal{\kappa}<\alpha_{n}<1$ for all $n$ and $\sum_{n=0}^{\infty}\left(\alpha_{n}-\kappa\right)\left(1-\alpha_{n}\right)=\infty$. In 1974, S. Ishikawa proved the following strong convergence theorem of pseudocontractive mapping.

Theorem 1.2 (see [9]). Let $C$ be a convex compact subset of a Hilbert space $H$, and let $T: C \rightarrow C$ be a Lipschitzian pseudocontractive mapping. For any $x_{1} \in C$, suppose that the sequence $\left\{x_{n}\right\}$ is defined by

$$
\begin{gathered}
y_{n}=\left(1-\beta_{n}\right) x_{n}+\beta_{n} T x_{n}, \\
x_{n+1}=\left(1-\alpha_{n}\right) x_{n}+\alpha_{n} T y_{n}, \quad \forall n \in \mathbb{N},
\end{gathered}
$$

where $\left\{\alpha_{n}\right\},\left\{\beta_{n}\right\}$ are two real sequences in $[0,1]$ satisfying

(i) $\alpha_{n} \leq \beta_{n}$, for all $n \in \mathbb{N}$,

(ii) $\lim _{n \rightarrow \infty} \beta_{n}=0$,

(iii) $\sum_{n=1}^{\infty} \alpha_{n} \beta_{n}=\infty$.

Then $\left\{x_{n}\right\}$ converges strongly to a fixed point of $T$.

In order to prove a strong convergence theorem of Mann algorithm (1.12) associated with strictly pseudocontractive mapping, in 2006, Marino and Xu [7] proved the following theorem for strict pseudocontractive mapping in Hilbert space by using $C Q$ method. 
Theorem 1.3 (see [7]). Let $C$ be a closed convex subset of a Hilbert space $H$. Let $T: C \rightarrow C$ be a $\kappa$-strict pseudocontraction for some $0 \leq \kappa<1$, and assume that the fixed point set $F(T)$ of $T$ is nonempty. Let $\left\{x_{n}\right\}_{n=1}^{\infty}$ be the sequence generated by the following $(C Q)$ algorithm:

$$
\begin{gathered}
x_{1} \in C, \\
y_{n}=\alpha_{n} x_{n}+\left(1-\alpha_{n}\right) T x_{n}, \\
C_{n}=\left\{z \in C:\left\|y_{n}-z\right\|^{2} \leq\left(1-\alpha_{n}\right)\left(\mathcal{\kappa}-\alpha_{n}\right)\left\|x_{n}-T x_{n}\right\|^{2}\right\}, \\
Q_{n}=\left\{z \in C:\left\langle x_{n}-z, x_{1}-x_{n}\right\rangle\right\}, \\
x_{n+1}=P_{C_{n} \cap Q_{n}} x_{1} .
\end{gathered}
$$

Assume that the control sequence $\left\{\alpha_{n}\right\}_{n=1}^{\infty}$ is chosen so that $\alpha_{n}<1$ for all $n \in \mathbb{N}$. Then $\left\{x_{n}\right\}$ converges strongly to $P_{F(T)} x_{1}$. Very recently, in 2010, [10] established the hybrid algorithm for Lipschitz pseudocontractive mapping as follows:

$$
\begin{gathered}
\text { For } C_{1}=C, \quad x_{1}=P_{C_{1}} x_{1}, \\
y_{n}=\left(1-\alpha_{n}\right) x_{n}+\alpha_{n} T z_{n}, \\
z_{n}=\left(1-\beta_{n}\right) x_{n}+\beta_{n} T x_{n}, \\
C_{n+1}=\left\{z \in C_{n}:\left\|\alpha_{n}(I-T) y_{n}\right\|^{2} \leq 2 \alpha_{n}\left\langle x_{n}-z,(I-T) y_{n}\right\rangle\right. \\
\left.+2 \alpha_{n} \beta_{n} L\left\|x_{n}-T x_{n}\right\|\left\|y_{n}-x_{n}+\alpha_{n}(I-T) y_{n}\right\|\right\}, \\
x_{n+1}=P_{C_{n+1}} x_{1}, \quad \forall n \in \mathbb{N} .
\end{gathered}
$$

Under suitable conditions of $\left\{\alpha_{n}\right\}$ and $\left\{\beta_{n}\right\}$, they proved that the sequence $\left\{x_{n}\right\}$ defined by (1.15) converges strongly to $P_{F(T)} x_{1}$.

Many authors study the problem for finding a common element of the set of fixed point problem and the set of equilibrium problem in Hilbert spaces, for instance, [2, 3, 11-15]. The motivation of (1.14), (1.15), and the research in this direction, we prove the strong convergence theorem for finding solution of the set of fixed points of strictly pseudocontractive mapping and two sets of generalized equilibrium problems by using the hybrid method.

\section{Preliminaries}

In order to prove our main results, we need the following lemmas. Let $C$ be closed convex subset of a real Hilbert space $H$, and let $P_{C}$ be the metric projection of $H$ onto $C$; that is, for $x \in H, P_{C} x$ satisfies the property

$$
\left\|x-P_{C} x\right\|=\min _{y \in C}\|x-y\|
$$

The following characterizes the projection $P_{C}$. 
Lemma 2.1 (see [5]). Given that $x \in H$ and $y \in C$, then $P_{C} x=y$ if and only if the following inequality holds:

$$
\langle x-y, y-z\rangle \geq 0, \quad \forall z \in C
$$

The following lemma is well known.

Lemma 2.2. Let $H$ be Hilbert space, and let $C$ be a nonempty closed convex subset of $H$. Let $T$ : $C \rightarrow C$ be $\kappa$-strictly pseudocontractive, then the fixed point set $F(T)$ of $T$ is closed and convex so that the projection $P_{F(T)}$ is well defined.

Lemma 2.3 ((demiclosedness principle) (see [16])). If $T$ is a $\boldsymbol{\kappa}$-strict pseudocontraction on closed convex subset $C$ of a real Hilbert space $H$, then $I-T$ is demiclosed at any point $y \in H$.

To solve the equilibrium problem for a bifunction $F: C \times C \rightarrow \mathbb{R}$, assume that $F$ satisfies the following conditions:

(A1) $F(x, x)=0$ for all $x \in C$,

(A2) $F$ is monotone, that is, $F(x, y)+F(y, x) \leq 0$, for all $x, y \in C$,

(A3) for all $x, y, z \in C$,

$$
\lim _{t \rightarrow 0^{+}} F(t z+(1-t) x, y) \leq F(x, y)
$$

(A4) for all $x \in C, y \mapsto F(x, y)$ is convex and lower semicontinuous.

The following lemma appears implicitly in [1].

Lemma 2.4 (see [1]). Let $C$ be a nonempty closed convex subset of $H$, and let $F$ be a bifunction of $C \times C$ into $\mathbb{R}$ satisfying $(A 1)-(A 4)$. Let $r>0$, and $x \in H$. Then, there exists $z \in C$ such that

$$
F(z, y)+\frac{1}{r}\langle y-z, z-x\rangle
$$

for all $x \in C$.

Lemma 2.5 (see [11]). Assume that $F: C \times C \rightarrow \mathbb{R}$ satisfies $(A 1)-(A 4)$. For $r>0$ and $x \in H$, define a mapping $T_{r}: H \rightarrow C$ as follows:

$$
T_{r}(x)=\left\{z \in C: F(z, y)+\frac{1}{r}\langle y-z, z-x\rangle \geq 0, \forall y \in C\right\}
$$

for all $z \in H$. Then, the following hold:

(1) $T_{r}$ is single-valued; 
(2) $T_{r}$ is firmly nonexpansive, that is,

$$
\left\|T_{r}(x)-T_{r}(y)\right\|^{2} \leq\left\langle T_{r}(x)-T_{r}(y), x-y\right\rangle, \quad \forall x, y \in H,
$$

(3) $F\left(T_{r}\right)=E P(F)$;

(4) $E P(F)$ is closed and convex.

Lemma 2.6 (see [17]). Let $C$ be a closed convex subset of $H$. Let $\left\{x_{n}\right\}$ be a sequence in $H$ and $u \in H$. Let $q=P_{C} u ;$ if $\left\{x_{n}\right\}$ is such that $\omega\left(x_{n}\right) \subset C$ and satisfy the condition

$$
\left\|x_{n}-u\right\| \leq\|u-q\|, \quad \forall n \in \mathbb{N},
$$

then $x_{n} \rightarrow q$, as $n \rightarrow \infty$.

Lemma 2.7 (see [7]). For a real Hilbert space $H$, the following identities hold: if $\left\{x_{n}\right\}$ is a sequence in $H$ weak convergence to $z$, then

$$
\limsup _{n \rightarrow \infty}\left\|x_{n}-y\right\|^{2}=\limsup _{n \rightarrow \infty}\left\|x_{n}-z\right\|^{2}+\|z-y\|^{2}
$$

for all $y \in H$.

\section{Main Result}

Theorem 3.1. Let $C$ be a nonempty closed convex subset of a Hilbert space $H$. Let $F$ and $G$ be bifunctions from $C \times C$ into $\mathbb{R}$ satisfying $\left(A_{1}\right)-\left(A_{4}\right)$, respectively. Let $A: C \rightarrow H$ be an $\alpha$-inverse strongly monotone mapping, and let $B: C \rightarrow H$ be a $\beta$-inverse strongly monotone mapping. Let $T: C \rightarrow C$ be a $\mathcal{\kappa}$-strict pseudocontraction mapping with $\mathfrak{F}=F(T) \cap E P(F, A) \cap E P(G, B) \neq \emptyset$. Let $\left\{x_{n}\right\}$ be a sequence generated by $x_{1} \in C=C_{1}$ and

$$
\begin{gathered}
F\left(u_{n}, u\right)+\left(A x_{n}, u-u_{n}\right)+\frac{1}{r_{n}}\left\langle u-u_{n}, u_{n}-x_{n}\right\rangle \geq 0, \quad \forall u \in C, \\
G\left(v_{n}, v\right)+\left(B x_{n}, v-v_{n}\right)+\frac{1}{s_{n}}\left\langle v-v_{n}, v_{n}-x_{n}\right\rangle \geq 0, \quad \forall v \in C, \\
z_{n}=\delta_{n} u_{n}+\left(1-\delta_{n}\right) v_{n}, \\
y_{n}=\alpha_{n} z_{n}+\left(1-\alpha_{n}\right) T z_{n}, \\
C_{n+1}=\left\{z \in C_{n}:\left\|y_{n}-z\right\| \leq\left\|x_{n}-z\right\|\right\}, \\
x_{n+1}=P_{C_{n+1}} x_{1}, \quad \forall n \geq 1,
\end{gathered}
$$

where $\left\{\alpha_{n}\right\}_{n=0}^{\infty}$ is sequence in $[0,1], r_{n} \in[a, b] \subset(0,2 \alpha)$, and $s_{n} \subset[c, d] \subset(0,2 \beta)$ satisfy the following conditions: 
Fixed Point Theory and Applications

(i) $\lim _{n \rightarrow \infty} \delta_{n}=\delta \in(0,1)$,

(ii) $0 \leq \kappa \leq \alpha_{n}<1$, for all $n \geq 1$.

Then $x_{n}$ converges strongly to $P_{\mathfrak{F}} x_{1}$.

Proof. First, we show that $\left(I-r_{n} A\right)$ is nonexpansive. Let $x, y \in C$. Since $A$ is $\alpha$-inverse strongly monotone mapping and $r_{n}<2 \alpha$, we have

$$
\begin{aligned}
\left\|\left(I-r_{n} A\right) x-\left(I-r_{n} A\right) y\right\|^{2} & =\left\|x-y-r_{n}(A x-A y)\right\|^{2} \\
& =\|x-y\|^{2}-2 r_{n}\langle x-y, A x-A y\rangle+r_{n}^{2}\|A x-A y\|^{2} \\
& \leq\|x-y\|^{2}-2 \alpha r_{n}\|A x-A y\|^{2}+r_{n}^{2}\|A x-A y\|^{2} \\
& =\|x-y\|^{2}+r_{n}\left(r_{n}-2 \alpha\right)\|A x-A y\|^{2} \\
& \leq\|x-y\|^{2} .
\end{aligned}
$$

Thus $\left(I-r_{n} A\right)$ is nonexpansive, so are $I-s_{n} B, T_{r_{n}}\left(I-r_{n} A\right)$, and $T_{s_{n}}\left(I-s_{n} B\right)$. Since

$$
F\left(u_{n}, u\right)+\left\langle A x_{n}, u-u_{n}\right\rangle+\frac{1}{r_{n}}\left\langle u-u_{n}, u_{n}-x_{n}\right\rangle \geq 0, \quad \forall u \in C,
$$

then we have

$$
F\left(u_{n}, u\right)+\frac{1}{r_{n}}\left\langle u-u_{n}, u_{n}-\left(I-r_{n} A\right) x_{n}\right\rangle \geq 0 .
$$

By Lemma 2.5, we have $u_{n}=T_{r_{n}}\left(I-r_{n} A\right) x_{n}$. By the same argument as above, we conclude that $v_{n}=T_{S_{n}}\left(I-s_{n} B\right) x_{n}$.

Let $z \in \mathfrak{F}$. Then $F(z, y)+\langle y-z, A z\rangle \geq 0$ and $G(z, y)+\langle y-z, B z\rangle \geq 0$. Hence

$$
\begin{aligned}
& F(z, y)+\frac{1}{r_{n}}\left\langle y-z, z-z+r_{n} A z\right\rangle \geq 0, \\
& G(z, y)+\frac{1}{s_{n}}\left\langle y-z, z-z+s_{n} B z\right\rangle \geq 0 .
\end{aligned}
$$

Again by Lemma 2.5, we have $z=T_{r_{n}}\left(z-r_{n} A z\right)=T_{S_{n}}\left(z-s_{n} B z\right)$. By nonexpansiveness of $T_{r_{n}}\left(I-r_{n} A\right)$ and $T_{S_{n}}\left(I-s_{n} B\right)$, we have

$$
\begin{aligned}
\left\|u_{n}-z\right\| & =\left\|T_{r_{n}}\left(I-r_{n} A\right) x_{n}-T_{r_{n}}\left(I-r_{n} A\right) z\right\| \\
& \leq\left\|x_{n}-z\right\|, \\
\left\|v_{n}-z\right\| & =\left\|T_{S_{n}}\left(I-s_{n} A\right) x_{n}-T_{S_{n}}\left(I-s_{n} A\right) z\right\| \\
& \leq\left\|x_{n}-z\right\| .
\end{aligned}
$$


By (3.6), we have

$$
\left\|z_{n}-z\right\| \leq\left\|x_{n}-z\right\|
$$

Next, we show that $C_{n}$ is closed and convex for every $n \in \mathbb{N}$. It is obvious that $C_{n}$ is closed. In fact, we know that, for $z \in C_{n}$,

$$
\left\|y_{n}-z\right\| \leq\left\|x_{n}-z\right\| \quad \text { is equivalent to }\left\|y_{n}-x_{n}\right\|^{2}+2\left\langle y_{n}-x_{n}, x_{n}-z\right\rangle \leq 0
$$

So, we have that for all $z_{1}, z_{2} \in C_{n}$ and $t \in(0,1)$, it follows that

$$
\begin{aligned}
\left\|y_{n}-x_{n}\right\|^{2}+ & 2\left\langle y_{n}-x_{n}, x_{n}-\left(t z_{1}+(1-t) z_{2}\right)\right\rangle \\
= & t\left(2\left\langle y_{n}-x_{n}, x_{n}-z_{1}\right\rangle+\left\|y_{n}-x_{n}\right\|^{2}\right) \\
& +(1-t)\left(2\left\langle y_{n}-x_{n}, x_{n}-z_{2}\right\rangle+\left\|y_{n}-x_{n}\right\|^{2}\right) \\
\leq & 0 .
\end{aligned}
$$

Then, we have that $C_{n}$ is convex. By Lemmas 2.5 and 2.2 , we conclude that $\mathfrak{F}$ is closed and convex. This implies that $P_{\mathfrak{F}}$ is well defined. Next, we show that $\mathfrak{F} \subset C_{n}$ for every $n \in \mathbb{N}$.

Taking $p \in \mathfrak{F}$, we have

$$
\begin{aligned}
\left\|y_{n}-p\right\|^{2}= & \left\|\alpha_{n}\left(z_{n}-p\right)+\left(1-\alpha_{n}\right)\left(T z_{n}-p\right)\right\|^{2} \\
= & \alpha_{n}\left\|z_{n}-p\right\|^{2}+\left(1-\alpha_{n}\right)\left\|T z_{n}-p\right\|^{2}-\alpha_{n}\left(1-\alpha_{n}\right)\left\|z_{n}-T z_{n}\right\|^{2} \\
\leq & \alpha_{n}\left\|z_{n}-p\right\|^{2}+\left(1-\alpha_{n}\right)\left(\left\|z_{n}-p\right\|^{2}+\kappa\left\|(I-T) z_{n}-(I-T) p\right\|^{2}\right) \\
& -\alpha_{n}\left(1-\alpha_{n}\right)\left\|z_{n}-T z_{n}\right\|^{2} \\
= & \alpha_{n}\left\|z_{n}-p\right\|^{2}+\left(1-\alpha_{n}\right)\left\|z_{n}-p\right\|^{2}+\kappa\left(1-\alpha_{n}\right)\left\|z_{n}-T z_{n}\right\|^{2} \\
& -\alpha_{n}\left(1-\alpha_{n}\right)\left\|z_{n}-T z_{n}\right\|^{2} \\
= & \left\|z_{n}-p\right\|^{2}+\left(\kappa-\alpha_{n}\right)\left(1-\alpha_{n}\right)\left\|z_{n}-T z_{n}\right\|^{2} \\
\leq & \left\|z_{n}-p\right\|^{2} \\
\leq & \left\|x_{n}-p\right\|^{2} .
\end{aligned}
$$

It follows that $p \in C_{n}$. Then, we have $\mathfrak{F} \subset C_{n}$, for all $n \in \mathbb{N}$. Since $x_{n}=P_{C_{n}} x_{1}$, for every $w \in C_{n}$, we have

$$
\left\|x_{n}-x_{1}\right\| \leq\left\|w-x_{1}\right\|, \quad \forall n \in \mathbb{N}
$$


In particular, we have

$$
\left\|x_{n}-x_{1}\right\| \leq\left\|P_{\mathfrak{F}} x_{1}-x_{1}\right\| .
$$

By (3.11), we have that $\left\{x_{n}\right\}$ is bounded, so are $\left\{u_{n}\right\},\left\{v_{n}\right\},\left\{z_{n}\right\},\left\{y_{n}\right\}$. Since $x_{n+1}=P_{C_{n+1}} x_{1} \in$ $C_{n+1} \subset C_{n}$ and $x_{n}=P_{C_{n}} x_{1}$, we have

$$
\begin{aligned}
0 & \leq\left\langle x_{1}-x_{n}, x_{n}-x_{n+1}\right\rangle \\
& =\left\langle x_{1}-x_{n}, x_{n}-x_{1}+x_{1}-x_{n+1}\right\rangle \\
& \leq-\left\|x_{n}-x_{1}\right\|^{2}+\left\|x_{n}-x_{1}\right\|\left\|x_{1}-x_{n+1}\right\| .
\end{aligned}
$$

It is implied that

$$
\left\|x_{n}-x_{1}\right\| \leq\left\|x_{n+1}-x_{1}\right\|
$$

Hence, we have that $\lim _{n \rightarrow \infty}\left\|x_{n}-x_{1}\right\|$ exists. Since

$$
\begin{aligned}
\left\|x_{n}-x_{n+1}\right\|^{2} & =\left\|x_{n}-x_{1}+x_{1}-x_{n+1}\right\|^{2} \\
& =\left\|x_{n}-x_{1}\right\|^{2}+2\left\langle x_{n}-x_{1}, x_{1}-x_{n+1}\right\rangle+\left\|x_{1}-x_{n+1}\right\|^{2} \\
& =\left\|x_{n}-x_{1}\right\|^{2}+2\left\langle x_{n}-x_{1}, x_{1}-x_{n}+x_{n}-x_{n+1}\right\rangle+\left\|x_{1}-x_{n+1}\right\|^{2} \\
& =\left\|x_{n}-x_{1}\right\|^{2}-2\left\|x_{n}-x_{1}\right\|^{2}+2\left\langle x_{n}-x_{1}, x_{n}-x_{n+1}\right\rangle+\left\|x_{1}-x_{n+1}\right\|^{2} \\
& \leq\left\|x_{1}-x_{n+1}\right\|^{2}-\left\|x_{n}-x_{1}\right\|^{2},
\end{aligned}
$$

it is implied that

$$
\lim _{n \rightarrow \infty}\left\|x_{n}-x_{n+1}\right\|=0
$$

Since $x_{n+1}=P_{C_{n+1}} x_{1} \in C_{n+1}$, we have

$$
\left\|y_{n}-x_{n+1}\right\| \leq\left\|x_{n}-x_{n+1}\right\|,
$$

And by (3.16), we have

$$
\lim _{n \rightarrow \infty}\left\|y_{n}-x_{n+1}\right\|=0
$$

Since

$$
\left\|y_{n}-x_{n}\right\| \leq\left\|y_{n}-x_{n+1}\right\|+\left\|x_{n+1}-x_{n}\right\|,
$$


by (3.16) and (3.18), we have

$$
\lim _{n \rightarrow \infty}\left\|y_{n}-x_{n}\right\|=0
$$

Next, we show that

$$
\lim _{n \rightarrow \infty}\left\|u_{n}-x_{n}\right\|=0, \quad \lim _{n \rightarrow \infty}\left\|v_{n}-x_{n}\right\|=0 .
$$

Let $p \in \mathfrak{F}$, by (3.10) and (3.7), we have

$$
\begin{aligned}
\left\|y_{n}-p\right\|^{2}= & \left\|\alpha_{n}\left(z_{n}-p\right)+\left(1-\alpha_{n}\right)\left(T z_{n}-p\right)\right\|^{2} \\
= & \alpha_{n}\left\|z_{n}-p\right\|^{2}+\left(1-\alpha_{n}\right)\left\|T z_{n}-p\right\|^{2}-\alpha_{n}\left(1-\alpha_{n}\right)\left\|z_{n}-T z_{n}\right\|^{2} \\
\leq & \alpha_{n}\left\|z_{n}-p\right\|^{2}+\left(1-\alpha_{n}\right)\left(\left\|z_{n}-p\right\|^{2}+\kappa\left\|(I-T) z_{n}-(I-T) p\right\|^{2}\right) \\
& -\alpha_{n}\left(1-\alpha_{n}\right)\left\|z_{n}-T z_{n}\right\|^{2} \\
= & \alpha_{n}\left\|z_{n}-p\right\|^{2}+\left(1-\alpha_{n}\right)\left\|z_{n}-p\right\|^{2}+\kappa\left(1-\alpha_{n}\right)\left\|z_{n}-T z_{n}\right\|^{2} \\
& -\alpha_{n}\left(1-\alpha_{n}\right)\left\|z_{n}-T z_{n}\right\|^{2} \\
= & \alpha_{n}\left\|z_{n}-p\right\|^{2}+\left(1-\alpha_{n}\right)\left\|z_{n}-p\right\|^{2}+\left(\kappa-\alpha_{n}\right)\left(1-\alpha_{n}\right)\left\|z_{n}-T z_{n}\right\|^{2} \\
\leq & \alpha_{n}\left\|x_{n}-p\right\|^{2}+\left(1-\alpha_{n}\right)\left\|z_{n}-p\right\|^{2} \\
\leq & \alpha_{n}\left\|x_{n}-p\right\|^{2}+\left(1-\alpha_{n}\right)\left(\delta_{n}\left\|u_{n}-p\right\|^{2}+\left(1-\delta_{n}\right)\left\|v_{n}-p\right\|^{2}\right) .
\end{aligned}
$$

Since $u_{n}=T_{r_{n}}\left(I-r_{n} A\right) x_{n}, p=T_{r_{n}}\left(I-r_{n} A\right) p$, we have

$$
\begin{aligned}
\left\|u_{n}-p\right\|^{2} & =\left\|T_{r_{n}}\left(I-r_{n} A\right) x_{n}-T_{r_{n}}\left(I-r_{n} A\right) p\right\|^{2} \\
& \leq\left\|\left(I-r_{n} A\right) x_{n}-\left(I-r_{n} A\right) p\right\|^{2} \\
& =\left\|x_{n}-r_{n} A x_{n}-p+r_{n} A p\right\|^{2} \\
& =\left\|x_{n}-p-r_{n}\left(A x_{n}-A p\right)\right\|^{2} \\
& =\left\|x_{n}-p\right\|^{2}+r_{n}^{2}\left\|A x_{n}-A p\right\|^{2}-2 r_{n}\left\langle x_{n}-p, A x_{n}-A p\right\rangle \\
& \leq\left\|x_{n}-p\right\|^{2}+r_{n}^{2}\left\|A x_{n}-A p\right\|^{2}-2 r_{n} \alpha\left\|A x_{n}-A p\right\|^{2} \\
& =\left\|x_{n}-p\right\|^{2}+r_{n}\left(r_{n}-2 \alpha\right)\left\|A x_{n}-A p\right\|^{2} .
\end{aligned}
$$


Since $v_{n}=T_{s_{n}}\left(I-s_{n} B\right) x_{n}, p=T_{s_{n}}\left(I-s_{n} B\right) p$, we have

$$
\begin{aligned}
\left\|v_{n}-p\right\|^{2} & =\left\|T_{s_{n}}\left(I-s_{n} B\right) x_{n}-T_{s_{n}}\left(I-s_{n} B\right) p\right\|^{2} \\
& \leq\left\|\left(I-s_{n} B\right) x_{n}-\left(I-s_{n} B\right) p\right\|^{2} \\
& =\left\|x_{n}-s_{n} B x_{n}-p+s_{n} B p\right\|^{2} \\
& =\left\|x_{n}-p-s_{n}\left(B x_{n}-B p\right)\right\|^{2} \\
& =\left\|x_{n}-p\right\|^{2}+s_{n}^{2}\left\|B x_{n}-B p\right\|^{2}-2 s_{n}\left\langle x_{n}-p, B x_{n}-B p\right\rangle \\
& \leq\left\|x_{n}-p\right\|^{2}+s_{n}^{2}\left\|B x_{n}-B p\right\|^{2}-2 s_{n} \beta\left\|B x_{n}-B p\right\|^{2} \\
& =\left\|x_{n}-p\right\|^{2}+s_{n}\left(s_{n}-2 \beta\right)\left\|B x_{n}-B p\right\|^{2} .
\end{aligned}
$$

Substituting (3.23) and (3.24) into (3.22),

$$
\begin{aligned}
\left\|y_{n}-p\right\|^{2} \leq & \alpha_{n}\left\|x_{n}-p\right\|^{2}+\left(1-\alpha_{n}\right)\left(\delta_{n}\left\|u_{n}-p\right\|^{2}+\left(1-\delta_{n}\right)\left\|v_{n}-p\right\|^{2}\right) \\
\leq & \alpha_{n}\left\|x_{n}-p\right\|^{2} \\
& +\left(1-\alpha_{n}\right)\left(\delta_{n}\left(\left\|x_{n}-p\right\|^{2}+r_{n}\left(r_{n}-2 \alpha\right)\left\|A x_{n}-A p\right\|^{2}\right)\right. \\
& \left.\quad+\left(1-\delta_{n}\right)\left(\left\|x_{n}-p\right\|^{2}+s_{n}\left(s_{n}-2 \beta\right)\left\|B x_{n}-B p\right\|^{2}\right)\right) \\
= & \alpha_{n}\left\|x_{n}-p\right\|^{2} \\
& +\left(1-\alpha_{n}\right)\left(\delta_{n}\left\|x_{n}-p\right\|^{2}+\delta_{n} r_{n}\left(r_{n}-2 \alpha\right)\left\|A x_{n}-A p\right\|^{2}\right. \\
& \left.\quad+\left(1-\delta_{n}\right)\left\|x_{n}-p\right\|^{2}+s_{n}\left(1-\delta_{n}\right)\left(s_{n}-2 \beta\right)\left\|B x_{n}-B p\right\|^{2}\right) \\
= & \alpha_{n}\left\|x_{n}-p\right\|^{2}+\left(1-\alpha_{n}\right) \\
& \times\left(\left\|x_{n}-p\right\|^{2}+\delta_{n} r_{n}\left(r_{n}-2 \alpha\right)\left\|A x_{n}-A p\right\|^{2}+s_{n}\left(1-\delta_{n}\right)\left(s_{n}-2 \beta\right)\left\|B x_{n}-B p\right\|^{2}\right) \\
= & \alpha_{n}\left\|x_{n}-p\right\|^{2}+\left(1-\alpha_{n}\right)\left\|x_{n}-p\right\|^{2}+\left(1-\alpha_{n}\right) \delta_{n} r_{n}\left(r_{n}-2 \alpha\right)\left\|A x_{n}-A p\right\|^{2} \\
& +s_{n}\left(1-\alpha_{n}\right)\left(1-\delta_{n}\right)\left(s_{n}-2 \beta\right)\left\|B x_{n}-B p\right\|^{2} \\
= & \left\|x_{n}-p\right\|^{2}+\left(1-\alpha_{n}\right) \delta_{n} r_{n}\left(r_{n}-2 \alpha\right)\left\|A x_{n}-A p\right\|^{2} \\
& +s_{n}\left(1-\alpha_{n}\right)\left(1-\delta_{n}\right)\left(s_{n}-2 \beta\right)\left\|B x_{n}-B p\right\|^{2} .
\end{aligned}
$$


It is implied that

$$
\begin{aligned}
\left(1-\alpha_{n}\right) \delta_{n} r_{n}\left(2 \alpha-r_{n}\right)\left\|A x_{n}-A p\right\|^{2} \leq & \left\|x_{n}-p\right\|^{2}-\left\|y_{n}-p\right\|^{2} \\
& +s_{n}\left(1-\alpha_{n}\right)\left(1-\delta_{n}\right)\left(s_{n}-2 \beta\right)\left\|B x_{n}-B p\right\|^{2} \\
\leq & \left(\left\|x_{n}-p\right\|+\left\|y_{n}-p\right\|\right)\left\|x_{n}-y_{n}\right\| .
\end{aligned}
$$

By (3.20) and condition (i), we have

$$
\lim _{n \rightarrow \infty}\left\|A x_{n}-A p\right\|=0 .
$$

By using the same method as (3.27), we have

$$
\lim _{n \rightarrow \infty}\left\|B x_{n}-B p\right\|=0 .
$$

By Lemma 2.5 and firm nonexpansiveness of $T_{r_{n}}$, we have

$$
\begin{aligned}
\left\|u_{n}-p\right\|^{2}= & \left\|T_{r_{n}}\left(I-r_{n} A\right) x_{n}-T_{r_{n}}\left(I-r_{n} A\right) p\right\|^{2} \\
\leq & \left\langle\left(I-r_{n} A\right) x_{n}-\left(I-r_{n} A\right) p, u_{n}-p\right\rangle \\
= & \frac{1}{2}\left(\left\|\left(I-r_{n} A\right) x_{n}-\left(I-r_{n} A\right) p\right\|^{2}+\left\|u_{n}-p\right\|^{2}\right. \\
& \left.\quad-\left\|\left(I-r_{n} A\right) x_{n}-\left(I-r_{n} A\right) p-\left(u_{n}-p\right)\right\|^{2}\right) \\
= & \frac{1}{2}\left(\left\|\left(I-r_{n} A\right) x_{n}-\left(I-r_{n} A\right) p\right\|^{2}+\left\|u_{n}-p\right\|^{2}\right. \\
& \left.\quad-\left\|x_{n}-u_{n}-r_{n}\left(A x_{n}-A p\right)\right\|^{2}\right) \\
= & \frac{1}{2}\left(\left\|\left(I-r_{n} A\right) x_{n}-\left(I-r_{n} A\right) p\right\|^{2}+\left\|u_{n}-p\right\|^{2}\right. \\
& \left.\quad-\left(\left\|x_{n}-u_{n}\right\|^{2}+r_{n}^{2}\left\|A x_{n}-A p\right\|^{2}-2 r_{n}\left\langle x_{n}-u_{n}, A x_{n}-A p\right\rangle\right)\right) \\
\leq & \frac{1}{2}\left(\left\|x_{n}-p\right\|^{2}+\left\|u_{n}-p\right\|^{2}-\left\|x_{n}-u_{n}\right\|^{2}-r_{n}^{2}\left\|A x_{n}-A p\right\|^{2}\right. \\
\quad & \left.+2 r_{n}\left\langle x_{n}-u_{n}, A x_{n}-A p\right\rangle\right) .
\end{aligned}
$$


By (3.29), it is implied that

$$
\begin{aligned}
\left\|u_{n}-p\right\|^{2} \leq & \left\|x_{n}-p\right\|^{2}-\left\|x_{n}-u_{n}\right\|^{2}-r_{n}^{2}\left\|A x_{n}-A p\right\|^{2} \\
& +2 r_{n}\left\langle x_{n}-u_{n}, A x_{n}-A p\right\rangle \\
\leq & \left\|x_{n}-p\right\|^{2}-\left\|x_{n}-u_{n}\right\|^{2}-r_{n}^{2}\left\|A x_{n}-A p\right\|^{2} \\
& +2 r_{n}\left\|x_{n}-u_{n}\right\|\left\|A x_{n}-A p\right\| .
\end{aligned}
$$

Again, by Lemma 2.5 and firm nonexpansiveness of $T_{S_{n}}$, we have

$$
\begin{aligned}
\left\|v_{n}-p\right\|^{2}= & \left\|T_{s_{n}}\left(I-s_{n} B\right) x_{n}-T_{s_{n}}\left(I-s_{n} A\right) p\right\|^{2} \\
\leq & \left\langle\left(I-s_{n} B\right) x_{n}-\left(I-s_{n} B\right) p, v_{n}-p\right\rangle \\
= & \frac{1}{2}\left(\left\|\left(I-s_{n} B\right) x_{n}-\left(I-s_{n} B\right) p\right\|^{2}+\left\|v_{n}-p\right\|^{2}\right. \\
& \left.\quad-\left\|\left(I-s_{n} B\right) x_{n}-\left(I-s_{n} B\right) p-\left(v_{n}-p\right)\right\|^{2}\right) \\
= & \frac{1}{2}\left(\left\|\left(I-s_{n} B\right) x_{n}-\left(I-s_{n} B\right) p\right\|^{2}+\left\|v_{n}-p\right\|^{2}-\left\|x_{n}-v_{n}-s_{n}\left(B x_{n}-B p\right)\right\|^{2}\right) \\
= & \frac{1}{2}\left(\left\|\left(I-s_{n} B\right) x_{n}-\left(I-s_{n} B\right) p\right\|^{2}+\left\|v_{n}-p\right\|^{2}\right. \\
& \left.\quad-\left(\left\|x_{n}-v_{n}\right\|^{2}+s_{n}^{2}\left\|B x_{n}-B p\right\|^{2}-2 s_{n}\left\langle x_{n}-v_{n}, B x_{n}-B p\right\rangle\right)\right) \\
\leq & \frac{1}{2}\left(\left\|x_{n}-p\right\|^{2}+\left\|v_{n}-p\right\|^{2}-\left\|x_{n}-v_{n}\right\|^{2}-s_{n}^{2}\left\|B x_{n}-B p\right\|^{2}\right. \\
& \left.+2 s_{n}\left\langle x_{n}-v_{n}, B x_{n}-B p\right\rangle\right) .
\end{aligned}
$$

By (3.31), it is implied that

$$
\begin{aligned}
\left\|v_{n}-p\right\|^{2} & \leq\left\|x_{n}-p\right\|^{2}-\left\|x_{n}-v_{n}\right\|^{2}-s_{n}^{2}\left\|B x_{n}-B p\right\|^{2}+2 s_{n}\left\langle x_{n}-v_{n}, B x_{n}-B p\right\rangle \\
& \leq\left\|x_{n}-p\right\|^{2}-\left\|x_{n}-v_{n}\right\|^{2}-s_{n}^{2}\left\|B x_{n}-B p\right\|^{2}+2 s_{n}\left\|x_{n}-v_{n}\right\|\left\|B x_{n}-B p\right\| .
\end{aligned}
$$


Substituting (3.30) and (3.32) into (3.22), we have

$$
\begin{aligned}
\left\|y_{n}-p\right\|^{2} \leq & \alpha_{n}\left\|x_{n}-p\right\|^{2}+\left(1-\alpha_{n}\right)\left(\delta_{n}\left\|u_{n}-p\right\|^{2}+\left(1-\delta_{n}\right)\left\|v_{n}-p\right\|^{2}\right) \\
\leq & \alpha_{n}\left\|x_{n}-p\right\|^{2}+\left(1-\alpha_{n}\right) \\
& \times\left(\delta_{n}\left(\left\|x_{n}-p\right\|^{2}-\left\|x_{n}-u_{n}\right\|^{2}-r_{n}^{2}\left\|A x_{n}-A p\right\|^{2}+2 r_{n}\left\|x_{n}-u_{n}\right\|\left\|A x_{n}-A p\right\|\right)\right. \\
& +\left(1-\delta_{n}\right)\left(\left\|x_{n}-p\right\|^{2}-\left\|x_{n}-v_{n}\right\|^{2}-s_{n}^{2}\left\|B x_{n}-B p\right\|^{2}\right. \\
& \left.\left.\quad+2 s_{n}\left\|x_{n}-v_{n}\right\|\left\|B x_{n}-B p\right\|\right)\right) \\
\leq & \left.\alpha_{n}\left\|x_{n}-p\right\|^{2} \quad+\left(1-\delta_{n}\right)\left(\left\|x_{n}-p\right\|^{2}-\left\|x_{n}-v_{n}\right\|^{2}+2 s_{n}\left\|x_{n}-v_{n}\right\|\left\|B x_{n}-B p\right\|\right)\right) \\
& +\left(1-\alpha_{n}\right)\left(\delta_{n}\left(\left\|x_{n}-p\right\|^{2}-\left\|x_{n}-u_{n}\right\|^{2}+2 r_{n}\left\|x_{n}-u_{n}\right\|\left\|A x_{n}-A p\right\|\right)\right. \\
= & \alpha_{n}\left\|x_{n}-p\right\|^{2} \\
& +\left(1-\alpha_{n}\right)\left(\delta_{n}\left\|x_{n}-p\right\|^{2}-\delta_{n}\left\|x_{n}-u_{n}\right\|^{2}+2 \delta_{n} r_{n}\left\|x_{n}-u_{n}\right\|\left\|A x_{n}-A p\right\|+\left(1-\delta_{n}\right)\right. \\
& \left.\quad \times\left\|x_{n}-p\right\|^{2}-\left(1-\delta_{n}\right)\left\|x_{n}-v_{n}\right\|^{2}+2\left(1-\delta_{n}\right) s_{n}\left\|x_{n}-v_{n}\right\|\left\|B x_{n}-B p\right\|\right) \\
& \quad+\quad\left(1-\alpha_{n}\right)\left(1-\delta_{n}\right)\left\|x_{n}-v_{n}\right\|^{2}+2\left(1-\delta_{n}\right) s_{n}\left\|x_{n}-v_{n}\right\|\left\|B x_{n}-B p\right\|, \\
= & \alpha_{n}\left\|x_{n}-p\right\|^{2}+\left(1-\alpha_{n}\right) \\
& \times\left(\left\|x_{n}-p\right\|^{2}-\delta_{n}\left\|x_{n}-u_{n}\right\|^{2}+2 \delta_{n} r_{n}\left\|x_{n}-u_{n}\right\|\left\|A x_{n}-A p\right\|-\left(1-\delta_{n}\right)\left\|x_{n}-v_{n}\right\|^{2}\right. \\
& \left.+2\left(1-\delta_{n}\right) s_{n}\left\|x_{n}-v_{n}\right\|\left\|B x_{n}-B p\right\|\right)
\end{aligned}
$$

which implies that

$$
\begin{aligned}
\left(1-\alpha_{n}\right) \delta_{n}\left\|x_{n}-u_{n}\right\|^{2} \leq & \left\|x_{n}-p\right\|^{2}-\left\|y_{n}-p\right\|^{2}+2 \delta_{n} r_{n}\left\|x_{n}-u_{n}\right\|\left\|A x_{n}-A p\right\| \\
& -\left(1-\alpha_{n}\right)\left(1-\delta_{n}\right)\left\|x_{n}-v_{n}\right\|^{2}+2\left(1-\delta_{n}\right) s_{n}\left\|x_{n}-v_{n}\right\|\left\|B x_{n}-B p\right\| \\
\leq & \left\|x_{n}-y_{n}\right\|\left(\left\|x_{n}-p\right\|+\left\|y_{n}-p\right\|\right)+2 \delta_{n} r_{n}\left\|x_{n}-u_{n}\right\|\left\|A x_{n}-A p\right\| \\
& -\left(1-\alpha_{n}\right)\left(1-\delta_{n}\right)\left\|x_{n}-v_{n}\right\|^{2}+2\left(1-\delta_{n}\right) s_{n}\left\|x_{n}-v_{n}\right\|\left\|B x_{n}-B p\right\| \\
\leq & \left\|x_{n}-y_{n}\right\|\left(\left\|x_{n}-p\right\|+\left\|y_{n}-p\right\|\right)+2 \delta_{n} r_{n}\left\|x_{n}-u_{n}\right\|\left\|A x_{n}-A p\right\| \\
& +2\left(1-\delta_{n}\right) s_{n}\left\|x_{n}-v_{n}\right\|\left\|B x_{n}-B p\right\|,
\end{aligned}
$$


and by (3.27), (3.28), (3.20), and conditions (i), (ii), we have

$$
\lim _{n \rightarrow \infty}\left\|x_{n}-u_{n}\right\|=0
$$

By using the same method as (3.35), we have

$$
\lim _{n \rightarrow \infty}\left\|x_{n}-v_{n}\right\|=0
$$

Since

$$
\left\|z_{n}-x_{n}\right\| \leq \delta_{n}\left\|u_{n}-x_{n}\right\|+\left(1-\delta_{n}\right)\left\|v_{n}-x_{n}\right\|,
$$

from (3.35), (3.36), and condition (i), we have

$$
\lim _{n \rightarrow \infty}\left\|z_{n}-x_{n}\right\|=0
$$

By (3.20) and (3.38), we have

$$
\lim _{n \rightarrow \infty}\left\|y_{n}-z_{n}\right\|=0
$$

Since

$$
y_{n}-z_{n}=\left(1-\alpha_{n}\right)\left(T z_{n}-z_{n}\right),
$$

from (3.39) and condition (ii), we have

$$
\lim _{n \rightarrow \infty}\left\|T z_{n}-z_{n}\right\|=0
$$

Let $\omega\left(x_{n}\right)$ be the set of all weaks $\omega$-limit of $\left\{x_{n}\right\}$. We will show that $\omega\left(x_{n}\right) \subset \mathfrak{F}$. Since $\left\{x_{n}\right\}$ is bounded, then $\omega\left(x_{n}\right) \neq \emptyset$. Letting $q \in \omega\left(x_{n}\right)$, there exists a subsequence $\left\{x_{n_{i}}\right\}$ of $\left\{x_{n}\right\}$ converging to $q$. By (3.35), we have $u_{n_{i}} \rightarrow q$ as $i \rightarrow \infty$. Since $u_{n}=T_{r_{n}}\left(I-r_{n} A\right) x_{n}$, for any $y \in C$, we have

$$
F\left(u_{n}, y\right)+\left\langle A x_{n}, y-u_{n}\right\rangle+\frac{1}{r_{n}}\left\langle y-u_{n}, u_{n}-x_{n}\right\rangle \geq 0
$$

From $(A 2)$, we have

$$
\left\langle A x_{n}, y-u_{n}\right\rangle+\frac{1}{r_{n}}\left\langle y-u_{n}, u_{n}-x_{n}\right\rangle \geq F\left(y, u_{n}\right) .
$$


This implies that

$$
\left\langle A x_{n_{i}}, y-u_{n_{i}}\right\rangle+\frac{1}{r_{n_{i}}}\left\langle y-u_{n_{i}}, u_{n_{i}}-x_{n_{i}}\right\rangle \geq F\left(y, u_{n_{i}}\right) .
$$

Put $z_{t}=t y+(1-t) q$ for all $t \in(0,1]$ and $y \in C$. Then, we have $z_{t} \in C$. So, from (3.44), we have

$$
\begin{aligned}
\left\langle z_{t}-u_{n_{i}}, A z_{t}\right\rangle & \geq\left\langle z_{t}-u_{n_{i}}, A z_{t}\right\rangle-\left\langle z_{t}-u_{n_{i}}, A x_{n_{i}}\right\rangle-\left\langle z_{t}-u_{n_{i}}, \frac{u_{n_{i}}-x_{n_{i}}}{r_{n_{i}}}\right\rangle+F\left(z_{t}, u_{n_{i}}\right) \\
& =\left\langle z_{t}-u_{n_{i}}, A z_{t}-A u_{n_{i}}\right\rangle+\left\langle z_{t}-u_{n_{i}}, A u_{n_{i}}-A x_{n_{i}}\right\rangle-\left\langle z_{t}-u_{n_{i}}, \frac{u_{n_{i}}-x_{n_{i}}}{r_{n_{i}}}\right\rangle \\
& +F\left(z_{t}, u_{n_{i}}\right) .
\end{aligned}
$$

Since $\left\|u_{n_{i}}-x_{n_{i}}\right\| \rightarrow 0$, we have $\left\|A u_{n_{i}}-A x_{n_{i}}\right\| \rightarrow 0$. Further, from monotonicity of $A$, we have $\left\langle z_{t}-u_{n_{i}}, A z_{t}-A u_{n_{i}}\right\rangle \geq 0$. So, we have

$$
\left\langle z_{t}-q, A z_{t}\right\rangle \geq F\left(z_{t}, q\right) \text { as } i \longrightarrow \infty \text {. }
$$

From $(A 1),(A 4)$, and (3.46), we also have

$$
\begin{aligned}
0 & =F\left(z_{t}, z_{t}\right) \leq t F\left(z_{t}, y\right)+(1-t) F\left(z_{t}, q\right) \\
& \leq t F\left(z_{t}, y\right)+(1-t)\left\langle z_{t}-q, A z_{t}\right\rangle \\
& =t F\left(z_{t}, y\right)+(1-t) t\left\langle y-q, A z_{t}\right\rangle .
\end{aligned}
$$

Thus

$$
0 \leq F\left(z_{t}, y\right)+(1-t)\left\langle y-q, A z_{t}\right\rangle
$$

Letting $t \rightarrow 0$, we have, for each $y \in C$,

$$
0 \leq F(q, y)+\langle y-q, A q\rangle \text {. }
$$

This implies that

$$
q \in \operatorname{EP}(F, A)
$$

From (3.36), we have $v_{n i} \rightarrow q$. Since $v_{n}=T_{s_{n}}\left(I-s_{n} B\right) x_{n}$, for any $y \in C$, we have

$$
G\left(v_{n}, y\right)+\left\langle B x_{n}, y-v_{n}\right\rangle+\frac{1}{s_{n}}\left\langle y-v_{n}, v_{n}-x_{n}\right\rangle \geq 0
$$


By using the same method as (3.50), we have

$$
q \in \operatorname{EP}(G, B)
$$

Since $x_{n_{i}} \rightarrow q$ as $i \rightarrow \infty$ and (3.38), we have $z_{n_{i}} \rightarrow q$ as $i \rightarrow \infty$. By Lemma 2.3, $I-T$ is demiclosed at zero, and by (3.41), we have

$$
q \in F(T)
$$

From (3.50), (3.52), and (3.53), we have $q \in \mathfrak{F}$. Hence $\omega\left(x_{n}\right) \subset \mathfrak{F}$. Therefore, by (3.12) and Lemma 2.6, we have that $\left\{x_{n}\right\}$ converges strongly to $P_{\mathfrak{F}} x_{1}$. The proof is completed.

\section{Applications}

By using our main result, we have the following results in Hilbert spaces.

Theorem 4.1. Let $C$ be a nonempty closed convex subset of a Hilbert space $H$. Let $F$ and $G$ be bifunctions from $C \times C$ into $\mathbb{R}$ satisfying $\left(A_{1}\right)-\left(A_{4}\right)$, respectively. Let $T: C \rightarrow C$ be a $\kappa$-strict pseudocontraction mapping with $\mathfrak{F}=F(T) \cap E P(F) \cap E P(G) \neq \emptyset$. Let $\left\{x_{n}\right\}$ be a sequence generated by $x_{1} \in C=C_{1}$ and

$$
\begin{gathered}
F\left(u_{n}, u\right)+\frac{1}{r_{n}}\left\langle u-u_{n}, u_{n}-x_{n}\right\rangle \geq 0, \quad \forall u \in C, \\
G\left(v_{n}, v\right)+\frac{1}{s_{n}}\left\langle v-v_{n}, v_{n}-x_{n}\right\rangle \geq 0, \quad \forall v \in C, \\
z_{n}=\delta_{n} u_{n}+\left(1-\delta_{n}\right) v_{n}, \\
y_{n}=\alpha_{n} z_{n}+\left(1-\alpha_{n}\right) T z_{n}, \\
C_{n+1}=\left\{z \in C_{n}:\left\|y_{n}-z\right\| \leq\left\|x_{n}-z\right\|\right\}, \\
x_{n+1}=P_{C_{n+1}} x_{1}, \quad \forall n \geq 1,
\end{gathered}
$$

where $\left\{\alpha_{n}\right\}_{n=0}^{\infty}$ is sequence in $[0,1], r_{n} \in[a, b]$, and $s_{n} \subset[c, d]$ satisfy the following conditions:

(i) $\lim _{n \rightarrow \infty} \delta_{n}=\delta \in(0,1)$,

(ii) $0 \leq \kappa \leq \alpha_{n}<1$, for all $n \geq 1$.

Then $x_{n}$ converges strongly to $P_{\mathfrak{F}} x_{1}$.

Proof. Putting $A \equiv B \equiv 0$ in Theorem 3.1, we have the desired conclusions. 
Theorem 4.2. Let $C$ be a nonempty closed convex subset of a Hilbert space $H$. Let $F$ be bifunctions from $C \times C$ into $\mathbb{R}$ satisfying $\left(A_{1}\right)-\left(A_{4}\right)$, respectively. Let $A: C \rightarrow H$ be an $\alpha$-inverse strongly monotone mapping, and let $\left\{x_{n}\right\}$ be a sequence generated by $x_{1} \in C=C_{1}$ and

$$
\begin{gathered}
F\left(u_{n}, u\right)+\left(A x_{n}, u-u_{n}\right)+\frac{1}{r_{n}}\left\langle u-u_{n}, u_{n}-x_{n}\right\rangle \geq 0, \quad \forall u \in C, \\
y_{n}=\alpha_{n} u_{n}+\left(1-\alpha_{n}\right) T u_{n}, \\
C_{n+1}=\left\{z \in C_{n}:\left\|y_{n}-z\right\| \leq\left\|x_{n}-z\right\|\right\}, \\
x_{n+1}=P_{C_{n+1}} x_{1}, \quad \forall n \geq 1
\end{gathered}
$$

where $\left\{\alpha_{n}\right\}_{n=0}^{\infty}$ is sequence in $[0,1], r_{n} \in[a, b] \subset(0,2 \alpha)$, and $0 \leq \kappa \leq \alpha_{n}<1$, for all $n \geq 1$. Then $x_{n}$ converges strongly to $P_{\mathfrak{F}} x_{1}$.

Proof. Putting $G \equiv F, A \equiv B$, and $u_{n}=v_{n}$, for all $n \geq 1$, in Theorem 3.1, we have the desired conclusions.

\section{References}

[1] E. Blum and W. Oettli, "From optimization and variational inequalities to equilibrium problems," Mathematics Student, vol. 63, no. 1-4, pp. 123-145, 1994.

[2] A. Moudafi and M. Théra, "Proximal and dynamical approaches to equilibrium problems," in Ill-Posed Variational Problems and Regularization Techniques (Trier, 1998), vol. 477 of Lecture Notes in Economics and Mathematical Systems, pp. 187-201, Springer, Berlin, Germany, 1999.

[3] S. Takahashi and W. Takahashi, "Strong convergence theorem for a generalized equilibrium problem and a nonexpansive mapping in a Hilbert space," Nonlinear Analysis: Theory, Methods $\mathcal{E}$ Applications, vol. 69 , no. 3, pp. 1025-1033, 2008.

[4] H. Iiduka and W. Takahashi, "Weak convergence theorems by Cesáro means for nonexpansive mappings and inverse-strongly-monotone mappings," Journal of Nonlinear and Convex Analysis, vol. 7, no. 1, pp. 105-113, 2006.

[5] W. Takahashi, Nonlinear Functional Analysis. Fixed Point Theory and Its Applications, Yokohama Publishers, Yokohama, Japan, 2000.

[6] F. E. Browder and W. V. Petryshyn, "Construction of fixed points of nonlinear mappings in Hilbert space," Journal of Mathematical Analysis and Applications, vol. 20, pp. 197-228, 1967.

[7] G. Marino and H.-K. Xu, "Weak and strong convergence theorems for strict pseudo-contractions in Hilbert spaces," Journal of Mathematical Analysis and Applications, vol. 329, no. 1, pp. 336-346, 2007.

[8] W. R. Mann, "Mean value methods in iteration," Proceedings of the American Mathematical Society, vol. 4 , pp. 506-510, 1953.

[9] S. Ishikawa, "Fixed points by a new iteration method," Proceedings of the American Mathematical Society, vol. 44, pp. 147-150, 1974.

[10] Y.-C. Tanga, J.-G. Penga, and L.-W. Liu, "Strong convergence theorem for pseudo-contractive mappings in Hilbert spaces," Nonlinear Analysis: Theory, Methods \& Applications, vol. 74, pp. 380-385, 2011.

[11] P. L. Combettes and S. A. Hirstoaga, "Equilibrium programming in Hilbert spaces," Journal of Nonlinear and Convex Analysis, vol. 6, no. 1, pp. 117-136, 2005.

[12] A. Kangtunyakarn and S. Suantai, "A new mapping for finding common solutions of equilibrium problems and fixed point problems of finite family of nonexpansive mappings," Nonlinear Analysis: Theory, Methods E Applications, vol. 71, no. 10, pp. 4448-4460, 2009.

[13] A. Kangtunyakarn and S. Suantai, "Hybrid iterative scheme for generalized equilibrium problems and fixed point problems of finite family of nonexpansive mappings," Nonlinear Analysis. Hybrid Systems, vol. 3, no. 3, pp. 296-309, 2009. 
[14] S. Takahashi and W. Takahashi, "Viscosity approximation methods for equilibrium problems and fixed point problems in Hilbert spaces," Journal of Mathematical Analysis and Applications, vol. 331, no. 1, pp. 506-515, 2007.

[15] V. Colao, G. Marino, and H.-K. Xu, "An iterative method for finding common solutions of equilibrium and fixed point problems," Journal of Mathematical Analysis and Applications, vol. 344, no. 1, pp. 340352, 2008.

[16] H. Zhou, "Convergence theorems of fixed points for -strict pseudo-contractions in Hilbert spaces," Nonlinear Analysis: Theory, Methods E Applications, vol. 69, no. 2, pp. 456-462, 2008.

[17] C. Martinez-Yanes and H.-K. Xu, "Strong convergence of the CQ method for fixed point iteration processes," Nonlinear Analysis: Theory, Methods E Applications, vol. 64, no. 11, pp. 2400-2411, 2006. 